\title{
Patient Selection for Stroke Endovascular Therapy- DWI-ASPECTS Thresholds Should Vary among Age Groups: Insights from the RECOST Study
}

F. Danière, K. Lobotesis, P. Machi, O. Eker, I. Mourand, C. Riquelme, X. Ayrignac, J.F. Vendrell, G. Gascou, J. Fendeleur, C. Dargazanli, R. Schaub, H. Brunel, C. Arquizan, A. Bonafé, and V. Costalat

\begin{abstract}
BACKGROUND AND PURPOSE: The purpose of this study was to evaluate the benefits of endovascular intervention in large-vessel occlusion strokes, depending on age class.
\end{abstract}

MATERIALS AND METHODS: A clinical management protocol including intravenous treatment and mechanical thrombectomy was instigated in our center in 2009 (Prognostic Factors Related to Clinical Outcome Following Thrombectomy in Ischemic Stroke [RECOST] study). All patients with acute ischemic stroke with an anterior circulation major-vessel occlusion who presented within 6 hours were evaluated with an initial MR imaging examination and were analyzed according to age subgroups (younger than 50 years, 50-59 years, $60-69$ years, $70-79$ years; 80 years or older). The mRS score at 3 months was the study end point.

RESULTS: One hundred sixty-five patients were included in the analysis. The mean age was 67.4 years (range, 29-90 years). The mean baseline NIHSS score was 17.24 (range, 3-27). The mean DWI-derived ASPECTS was 6.4. Recanalization of TICI 2b/3 was achieved in $80 \%$. At 3 months, $41.72 \%$ of patients had a good outcome, with a gradation of prognosis depending on the age subgroup and a clear cutoff at 70 years. Only $19 \%$ of patients older than 80 years had a good outcome at 3 months (mean ASPECTS $=7.4$ ) with $28 \%$ for $70-79$ years (mean ASPECTS $=6.8$ ), but $58 \%$ for $60-69$ years (mean ASPECTS = 6), 52\% for 50-59 years (mean ASPECTS $=5.91$ ), and $72 \%$ for younger than 50 years (mean ASPECTS = 6.31). In contrast, the mortality rate was $35 \%$ for 80 years and older, and $26 \%$ for $70-79$ versus $5 \%-9 \%$ for younger than 70 years.

CONCLUSIONS: The elderly may benefit from thrombectomy when their ischemic core volume is low in comparison with younger patients who still benefit from acute recanalization despite larger infarcts. Stroke volume thresholds should, therefore, be related and adjusted to the patient's age group.

I schemic stroke is the third leading cause of death in France (fourth in the United States ${ }^{1,2}$ ), leading to significant disability. ${ }^{3}$ The World Health Organization predicts an increasing number of strokes in Europe during the next 10 years. ${ }^{4}$ Intravenous recombinant tissue plasminogen activator for cerebral arterial occlusion is the established therapy to date for acute ischemic stroke. The odds ratio benefit is $1.28-1.7$ for a favorable outcome versus

Received October 31, 2013; accepted after revision June 23, 2014. From the Departments of Neuroradiology (F.D., P.M., O.E., C.R., J.F.V., G.G., C.D., A.B., V.C.), Neurology (I.M., X.A., C.A.), and Anesthesiology (J.F.), CHU Montpellier, Montpellier, France; Imaging Department (K.L.), Imperial College Healthcare National Health Service Trust, Charing Cross Hospital, London, United Kingdom; Department of Medical Statistics (R.S.), CHU Montpellier, Arnaud de Villeneuve Hospital, University of Montpellier, Montpellier, France; and Department of Neuroradiology (H.B.), CHU Marseille, Hôpital La Timone, Marseille, France.

Please address correspondence to Vincent Costalat, PhD, CHU Montpellier, Neuroradiology, Avenue Augustin Fliche, Montpellier, France; e-mail: v-costalat@ chu-montpellier.fr

三 Indicates article with supplemental on-line table

http://dx.doi.org/10.3174/ajnr.A4104 a placebo ${ }^{5,6}$ within 4.5 hours after stroke onset. Initially, being older than 80 years of age was established as a bad prognostic factor in intravenous treatment ${ }^{7-10}$ and even considered an exclusion criterion. ${ }^{5}$ Today, this statement is seen as controversial, with recent studies having shown that elderly patients would still benefit from IV tPA. ${ }^{11,12}$ In addition, new therapeutic strategies in stroke units are increasingly involving adjunctive endovascular techniques when fibrinolysis is contraindicated or has failed or when large and proximal intracranial vessels are occluded. ${ }^{13-17}$ Previous studies have shown that mechanical thrombectomy by using Stentrievers (Trevo; Stryker, Kalamazoo, Michigan), in particular new stent retrievers, ${ }^{18}$ was successful in achieving a high rate of arterial recanalization and favorable clinical outcome in large-vessel occlusion. ${ }^{19-21}$ Nevertheless, inclusion and exclusion criteria for these new invasive strategies are still being evaluated and may need to be optimized to avoid futile recanalization, particularly for fragile patients. The purpose of this study was to investigate the benefits and safety of these new recanalization devices according to age subgroups. 


\section{MATERIALS AND METHODS \\ Data Selection}

From September 2009 to May 2012, a management protocol including intravenous therapy and mechanical thrombectomy was instigated in our center RECOST study, which followed discussion and consensus agreement among our neurologists, neuroradiologists, and anesthetists. Prospective data collection via a specific stroke data base was organized under the supervision of dedicated clinical research assistance. Acceptance by the institutional ethics committee of Montpellier-Centre Hospitalier Universitaire was also obtained. Only anterior circulation strokes were retrieved from this data base to select a more homogeneous stroke population. At the time of the study, 211 patients had been entered and 165 were retrieved for analysis, from which 151 had completed follow-up assessment at 3 months. Patients were divided following age subgroups: younger than 50 years, between 50 and 59 years, between 60 and 69 years, between 70 and 79 years, and older than 80 years.

\section{Initial Neurologic Examination}

At first, a stroke neurologist systematically evaluated all patients with the suspicion of an acute ischemic stroke and assessed the severity of the neurologic deficit by using the National Institutes of Health Stroke Scale score.

\section{MR Imaging Protocol}

A comprehensive acute brain MR imaging examination with MRA was performed as part of the initial patient work-up. MR imaging examinations were performed by using a $1.5 \mathrm{~T}$ wholebody MR imaging system (Magneton Avanto; Siemens, Erlangen, Germany), equipped with a standard 16-channel receive-only head-neck coil. Standardized imaging protocol covering the entire brain included an axial isotropic diffusion-weighted echoplanar spin-echo sequence with $b=0$ and $b=1000 \mathrm{~s} / \mathrm{mm}^{2}$ (TR/TE, 4900/90 ms; 25 sections; section thickness, $5 \mathrm{~mm}$; gap, $0 \mathrm{~mm}$; voxel size, $1.9 \times 1.9 \times 5 \mathrm{~mm}^{3}$; acquisition time, 44 seconds), axial T2 ${ }^{\star}$-weighted gradient-echo sequences, fluid-attenuated inversion-recovery sequences, and axial T2. T2 gradient-echo imaging was performed to screen for intracranial hemorrhage. DWI sequences were acquired, as was an apparent diffusion coefficient map to identify the infarct core. The Alberta Stroke Program Early CT Score, calculated on DWI, was used to assess and quantify the infarct core. ${ }^{22,23}$ FLAIR and T2 were also used to corroborate information regarding the time from symptom onset. FLAIR can assess slow flow in the distal territory, showing bright vessels, which are associated with good outcome after recanalization with IV treatment. Parenchymal FLAIR hypersignal was used by the neurologic team to decide on IV treatment but did not interfere with the decision-making for mechanical treatment. When the FLAIR findings were negative, the T2 was cancelled to shorten the examination. When they were positive, the T2 was performed to confirm a physiologic aging of the infarct suspected on the FLAIR. Then, a high-spatial-resolution 3D contrast-enhanced MR angiography with centric $k$-space ordering was performed just before the perfusion imaging with a fast-spoiled gradient refocused-echo sequence in the coronal plane: (TR/TE, 3.44/1.2 ms; flip angle, $28^{\circ}$; FOV, $300 \times 197 \mathrm{~mm}$; voxel size, $1 \times 0.8 \times 0.7$ $\mathrm{mm}^{3}$ ). One hundred four sections with a thickness of $0.7 \mathrm{~mm}$ in the coronal plane, providing arterial coverage from the aortic arch to the cranial vertex, were obtained during a 22 -second acquisition. A phase-contrast solution $(0.2 \mathrm{~mL} / \mathrm{kg}, 0.5-\mathrm{mmol} / \mathrm{kg}$ GdDTPA) was then injected and flushed with $20 \mathrm{~mL}$ of saline at 2 $\mathrm{mL} / \mathrm{s}$ for the contrast-enhanced MRA acquisition. It depicts intracranial and extracranial large-vessel occlusion. If the clinical/ radiologic mismatch was not clear, a gradient-echo echo-planar imaging sequence (TR/TE, 1420/25 ms; 25 sections; section thickness, $5 \mathrm{~mm}$; gap, $0 \mathrm{~mm}$; pixel size, $1.8 \times 1.8 \times 5 \mathrm{~mm}^{3}$; 65 dynamic frames at intervals of 2 seconds) was performed synchronized with an intravenous contrast agent injection (0.5-mmol/kg Gd-DTPA) with a flow rate of $6 \mathrm{~mL} / \mathrm{s}$ followed by a flush of $20-\mathrm{mL}$ saline. These perfusion-weighted images were used to assess a diffusion/perfusion mismatch. If the clinical/radiologic mismatch appeared to be selfevident (ie, an ASPECTS 8 with an NIHSS score of 18), no perfusion was mandatory because the NIHSS score was considered the main biomarker of impaired brain perfusion.

The mean time between MR imaging door entrance and MR imaging ending was 22 minutes. Furthermore, patients with wake-up stroke are treated routinely in our center on the basis of the FLAIR sequences. Since 2009, the mechanical recanalization in our clinical center follows MR imaging selection. A second neuroradiologist not involved in the initial management reviewed all MR imaging.

\section{Stroke Management Protocol}

Inclusion criteria for endovascular therapy were the following: proximal major vessel occlusion (middle cerebral artery: proximal M1, distal M1, M1-M2, M2; intracranial carotid bifurcation; tandem M1 with an ICA occlusion), all patients with stroke with a relevant deficit, and a time window of $<6$ hours. Exclusion criteria were the following: MR imaging-ASPECTS $<5$; NIHSS of $<8$; and spontaneous NIHSS improvement. The FLAIR/T2 images were not considered exclusion criteria for mechanical treatment.

An ASPECTS $\geq 5$ on DWI sequences and an NIHSS of $\geq 8$ were the only criteria of inclusion written down for the protocol. Nevertheless, the interventionist and neurologist were systematically checking for a clinical/radiologic mismatch between DWI and NIHSS to confirm their decision. If unclear (ie, ASPECTS 5 and NIHSS 9), the PWI/DWI mismatch was studied to confirm the presence of salvageable brain based on the TTP map, with a threshold set at +5 seconds (Fig 1). ${ }^{24,25}$ The volume and the topography of the penumbra were analyzed by the neuroradiologist regarding the volume ratio (PWI/DWI) and the functional importance of the cortical area involved in this expected salvageable brain. The main goal was to avoid any futile recanalization.

For all patients with ischemic stroke presenting with a largevessel occlusion confirmed on MR imaging, 2 therapeutic options were proposed, depending on the time from symptom onset and contraindications to intravenous thrombolysis. Stand-alone thrombectomy was performed in patients who were outside the IV fibrinolytic therapeutic window (ie, between 4.5 and 6 hours $)^{26,27}$ and in patients with any contraindications to IV fibrinolysis, (eg, warfarin, anticoagulation therapy with international normalized ratio of $>1.5$, recent surgery, or history of hemorrhage/hematoma). A combined therapy, including IV fibrinolysis 


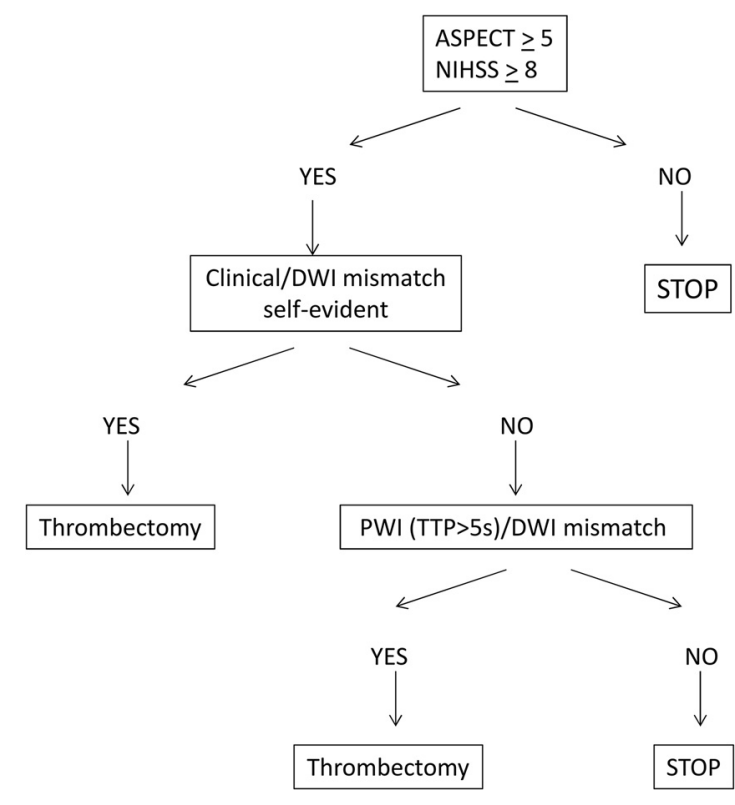

FIG 1. Use of DWI/PWI mismatch in patient selection.

$(0.9 \mathrm{mg} / \mathrm{kg})$ and thrombectomy, was chosen in all other cases within 4.5 hours after symptom onset.

\section{Device}

Endovascular procedures were performed by using several stent retrievers (Solitaire FR; Covidien, Irvine, California; Revive; Codman Neurovascular, Raynham, Massachusetts; Trevo). These devices are a stent-based thrombectomy system with a closed-cell design and a longitudinal split section. They are delivered through a standard microcatheter (inner lumen diameter of 0.021 inches or 0.027 inches) via a 0.016 -inch nitinol push wire.

\section{Mechanical Thrombectomy Protocol}

After MR imaging, the anesthesiologist followed the patient with stroke to the angiosuite and performed a fast induction while the interventionist was preparing the table. This general anesthesia did not delay the routine management and offered very quick access to the target vessel without patient motion. All procedures were performed via a femoral artery approach. An 8F or 9F Merci balloon guiding catheter (Concentric Medical, Mountain View, California) was introduced through a femoral sheath into the appropriate carotid artery. A 0.21-inch-internal-diameter microcatheter, Prowler Select Plus (Codman \& Shurtleff, Raynham, Massachusetts), Vasco 21 (Balt, Montmorency, France), or Headway microcatheter (MicroVention, Tustin, California), was navigated distal to the point of occlusion over a 0.014-inch steerable microwire. The stent retriever was then introduced through the microcatheter, and the device was deployed across the occluding thrombus and maintained in place for a few minutes (3-7 minutes). After this time, the fully deployed stent retriever and the delivery microcatheter were gently pulled back together and recovered through the balloon guiding catheter. Manual aspiration was performed through the hemostatic valve during the retrieval to reverse the flow and to aspirate clot debris eventually present in the lumen of the guide catheter. The integrity of the stent was consistently checked after every pass.
In our protocol, the patient received a full dose of IV fibrinolytics $(0.9 \mathrm{mg} / \mathrm{kg})$ when administered as a combined treatment. No additional IA fibrinolytics were allowed in the protocol to decrease the hemorrhagic-transformation risk. ${ }^{28}$

Recanalization was quantified by the Thrombolysis In Cerebral Infarction scale. ${ }^{29}$ Successful recanalization was defined as TICI $2 \mathrm{~b}$ or 3 in all treatable vessels. If the treatable vessel was not opened to at least TICI $2 b$ with a maximum of 5 passes of the thrombectomy device, then the treatment was considered failed. All TICI scores were independently reviewed by a second neuroradiologist not involved in the procedure.

\section{Postoperative Management}

CT was carried out in the angiogsuite after all procedures were performed, to screen for immediate hemorrhagic transformation, any unforeseen procedural complications, or initial blood-brain barrier disruption, which was defined as an intraparenchymal enhancement on a noncontrast CT, just after the mechanical treatment. A further CT or MR imaging was performed at 24 hours. All 24-hour CT and MR imaging was reviewed by a second neuroradiologist.

\section{Three-Month Follow-Up}

All patients were scheduled for a 3-month evaluation with a stroke neurologist. The modified Rankin Scale score at 3 months was recorded $^{30,31}$ and dichotomized as a favorable outcome with a score of $0-2$ or an unfavorable outcome with a score of 3-6.

\section{Statistical Analysis}

For univariate analysis, a global description of the sample was realized by giving the frequencies for categoric variables. Quantitative variables were described with means and SDs, but also with median, minimum, maximum, and interquartiles (25th and 75th centiles) if the distributions were not normal (tested with the Shapiro-Wilk test). For continuous variables, the comparisons of means between groups (good Rankin versus bad Rankin) were performed by using a parametric test (Student $t$ test) or a nonparametric test (Mann-Whitney $U$ test) if the distributions were not normal. For categoric variables, the comparisons of percentages between groups (good Rankin versus bad Rankin) were made by using the $\chi^{2}$ test or the Fisher exact test if the $\chi^{2}$ was not valid.

For multivariate analysis, the variables included in the logistic model were those that have been shown previously to be associated with studied dependent variables. To determine the relative importance of the predictor variables with regard to the modified Rankin Scale score, we performed multivariate analysis by using logistic regression. A stepwise selection of the variables was used. Age, NIHSS score on arrival, and duration of the obstruction were entered into the model as continuous variables. The categoric variables entered in the model were dichotomous. Odds ratios and their confidence intervals were calculated. The $\alpha$-to-enter and $\alpha$-to-exit were set at .05. To assess the predictive ability of the model, we calculated the concordance rate between predicted and observed responses. The goodness of fit of the logistic regression model was assessed by using the likelihood ratio test and the Hosmer-Lemeshow test. All analyses were 2-tailed, with a $P$ value $<$ .05 considered statistically significant. Statistical analysis was per- 
formed by using SAS statistical software, Version 9 (SAS Institute, Cary, North Carolina).

\section{RESULTS}

\section{Patient Characteristics}

From September 2009 to May 2012, 211 consecutive patients with large-vessel occlusions were treated by mechanical thrombectomy in the setting of acute ischemic stroke (On-line Table). One hundred sixty-five of 211 patients who had an anterior circulation stroke (47\% women and 53\% men) were retrieved from the data base. The mean age was 67.4 years (range, 29-90 years). The mean baseline NIHSS was 17.24 (range, 3-27) with higher initial NIHSS in the elderly: 16.39 (younger than 50 years), 15.24 (50-59 years), 16.54 ( $60-69$ years), 17.84 ( $70-79$ years), and 18.83 ( 80 years and older).

\section{Acute MR Imaging}

The mean ASPECTS on DWI was 6.40: 6.39 (younger than 50 years), 6.27 (50-59 years), 5.77 (60-69 years), 6.43 (70-79 years), and 7.25 (80 years and older) (On-line Table). Sixteen patients had an intracranial carotid bifurcation occlusion $(9.7 \%)$; 49, a tandem occlusion (29.7\%); 93, an M1 occlusion (56.36\%); and 7, an M2 occlusion (4.24\%). MR perfusion was performed in 38 cases and showed a perfusion-diffusion mismatch in 33 cases (87\%).

\section{Recanalization Rate}

Fifty-five patients $(33.0 \%)$ received stand-alone thrombectomy, and 110 patients $(67.0 \%)$ received a combined therapy, less frequent in those 80 years of age and older (On-line Table). Recanalization of TICI 3 was achieved in 59.76\% (98 patients); and TICI 2b, in $20.12 \%$ (33 patients), with an overall successful recanalization rate of $80 \%$ (TICI 3 and TICI $2 \mathrm{~b}$ ) with no differences among age groups. A failure rate was observed in $20 \%$. The mean time between symptom onset and recanalization was 311 minutes (range, 153-574 minutes) with a mean number of stent retriever passes of 2 without differences among age groups.

\section{Peri- and Postprocedural Complications}

There were 27 (16.46\%) periprocedural complications: 17 distal or collateral emboli, 5 dissections, 2 arterial injuries, and 3 spasms (On-line Table). These were without significant differences among ages: $11 \%$ (younger than 50 years), 22\% (50-59 years), $22 \%$ (60-69 years), 26\% (70-79 years), and 19\% ( 80 years and older). Initial blood-brain barrier disruption was found in $87 \mathrm{pa}-$ tients $(62.59 \%)$. Intracranial hemorrhagic transformation on day 1 on CT or MR imaging was depicted in 41 patients (25.95\%) and was symptomatic in only $7.6 \%$, but it was not correlated to the recanalization rate. Ischemic lesion worsening on day 1 on CT or MR imaging was found in 38 patients (25\%). There was a significant increase in ischemic lesion worsening for patients who did not experience recanalization (58\%) versus those who did (17\%), $(P<.05)$.

\section{Clinical Follow-Up}

At 3 months, 14 patients were lost to follow-up (8.5\%), while $41.72 \%$ of patients had a good outcome (On-line Table). A clear

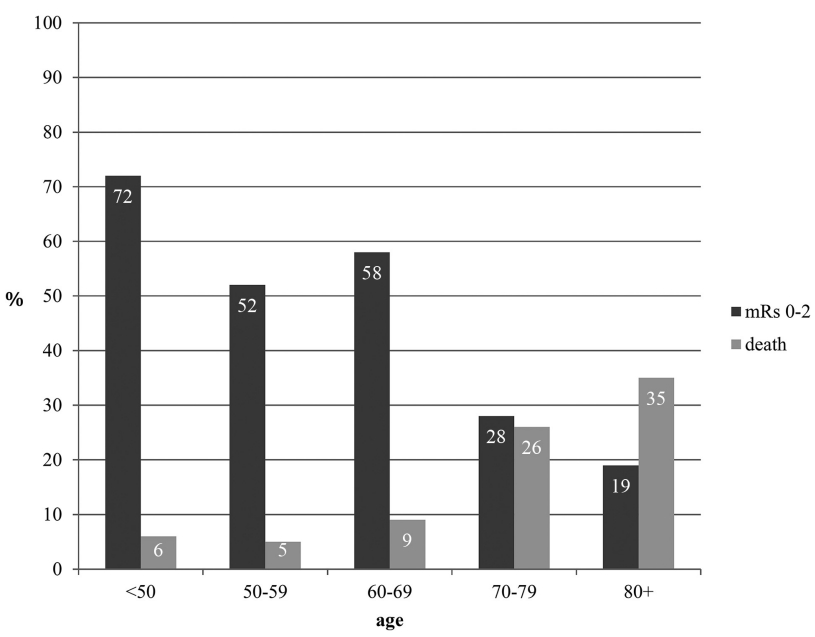

FIG 2. Modified Rankin Scale score and mortality according to the age subgroups.

Table 1: Univariate analysis

\begin{tabular}{|c|c|c|c|c|c|c|}
\hline Variable (mRS) & No. & Mean & Minimum & Median & Maximum & $\begin{array}{c}P \\
\text { Value }\end{array}$ \\
\hline \multicolumn{7}{|l|}{ Age } \\
\hline $0 / 1 / 2$ & 63 & 61.49 & 30.00 & 63.00 & 88.00 & $<.001$ \\
\hline $3 / 4 / 5 / 6$ & 88 & 71.32 & 29.00 & 75.00 & 90.00 & \\
\hline \multicolumn{7}{|l|}{ Initial NIHSS score } \\
\hline $0 / 1 / 2$ & 62 & 15.74 & 3.00 & 16.00 & 27.00 & $<.001$ \\
\hline $3 / 4 / 5 / 6$ & 86 & 18.21 & 6.00 & 19.00 & 27.00 & \\
\hline \multicolumn{7}{|l|}{ Initial ASPECTS } \\
\hline $0 / 1 / 2$ & 63 & 6.35 & 1.00 & 7.00 & 9.00 & .599 \\
\hline $3 / 4 / 5 / 6$ & 84 & 6.50 & 0.00 & 7.00 & 10.00 & \\
\hline \multicolumn{7}{|l|}{ Time } \\
\hline $0 / 1 / 2$ & 51 & 285.67 & 153.00 & 282.00 & 458.00 & .013 \\
\hline $3 / 4 / 5 / 6$ & 64 & 335.50 & 179.00 & 321.00 & 855.00 & \\
\hline \multicolumn{7}{|l|}{ Passes } \\
\hline $0 / 1 / 2$ & 63 & 1.63 & 0.00 & 1.00 & 5.00 & .005 \\
\hline $3 / 4 / 5 / 6$ & 87 & 2.15 & 0.00 & 2.00 & 5.00 & \\
\hline
\end{tabular}

gradation of the prognosis was observed depending on age, with a cutoff at 70 years (Fig 2). The mean age of patients with a good outcome was 61.5 years of age (95\% CI, 57.97-65.01) and 71.3 years of age (95\% CI, 68.71-73.93) in cases of bad outcome $(P<$ .001 ) (Table 1). Only $19 \%$ of patients who were older than 80 years (28\% for $70-79$ years) had a good outcome at 3 months (mRS $0-2$ ), with a $35 \%$ mortality rate ( $26 \%$ for $70-79$ years). On the contrary, good outcome was observed between $52 \%$ and $78 \%$ of patients younger than 70 years; mortality, in $<10 \%$ of patients (Fig 2). The mean modified Rankin Scale score at 3 months was 2.22 for patients younger than 70 years and 4.1 for those older than 70 years. On multivariate analysis, the odds ratio of bad outcomes was 1.092 (95\% CI, 1.046-1.141) for every 5 years' increase in age $(P<.0001)$ (Table 2$)$. The NIHSS were higher in those older than 70 years (18.1 versus $16.1 ; P=.007$ ). There were no other statistically significant differences comparing patients older than 70 years with those younger than 70 years in terms of sex, ASPECTS, recanalization rate, site of occlusion, time from onset to recanalization, or complications.

On univariate analysis, criteria significantly related to a good outcome at 3 months were initial NIHSS and rapid recanalization (Tables 1 and 3). The mean initial NIHSS related to good outcome was 15.74 versus 18.21 for bad outcome $(P<.001)$. A faster recanalization time was associated with a good outcome 
Table 2: Multivariate analysis

\begin{tabular}{lccc}
\hline \multicolumn{1}{c}{ Variable } & Adjusted Odds Ratio & $95 \% \mathrm{Cl}$ & $\boldsymbol{P}$ Value \\
\hline $\begin{array}{l}\text { Age } \\
\text { Each 5-yr increase }\end{array}$ & $1.092^{\mathrm{a}}$ & $1.046-1.141$ & $<.0001$ \\
$\begin{array}{l}\text { Time } \\
\text { Each 60-min increase }\end{array}$ & $1.009^{\mathrm{b}}$ & $1.002-1.016$ & .0093 \\
\hline
\end{tabular}

a For each 5-year increase, risk of bad outcomes at 3 months increased by 1.092 .

${ }^{b}$ For each 60-minute increase to recanalization time, bad outcomes at 3 months increased by 1.009 .

Table 3: Univariate analysis

\begin{tabular}{lrrr}
\hline \multicolumn{1}{c}{ Variable } & mRS 0-2 & mRS 3-6 & P Value \\
\hline TICl score & & & \\
O/1/2a & $13.33 \%$ & $86.67 \%$ & .000329 \\
2b/3 & $49.58 \%$ & $50.42 \%$ & \\
BBBD & & & \\
$\quad$ No & $50.98 \%$ & $49.02 \%$ & .095777 \\
Yes & $36.25 \%$ & $63.75 \%$ & \\
Aggravation of lesions & & & \\
$\quad$ No & $53.33 \%$ & $46.67 \%$ & .000021 \\
$\quad$ Yes & $11.76 \%$ & $88.24 \%$ & \\
Hemorrhagic or complications at $24 \mathrm{hr}$ & & & \\
$\quad$ No & $49.06 \%$ & $50.94 \%$ & .002663 \\
$\quad$ Yes & $21.05 \%$ & $78.95 \%$ & \\
\hline
\end{tabular}

Note:-BBBD indicates blood-brain barrier disruption.

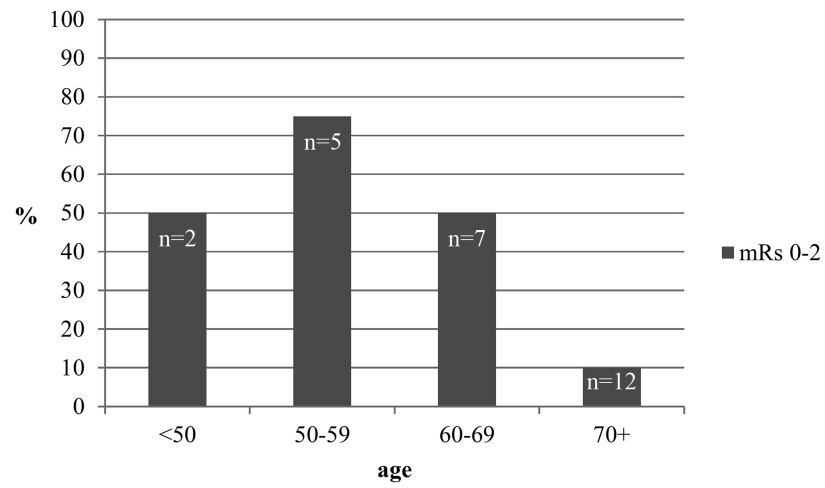

FIG 3. Outcome in protocol deviation (DWI-ASPECTS $<5$ ) according to the age subgroups.

(mean time between onset and recanalization, 286 minutes and 7 seconds; $P=.013)$. The number of stent retriever passes was also correlated to clinical outcome with 1.63 passes for good outcome and 2.15 for bad outcome $(P=.005)$. The final recanalization score of TICI $0 / 1 / 2$ a was a strong predictive factor for a bad outcome with $87 \%$ with mRS $3-6(P<.0003)$ at 3 months. Hemorrhagic transformation and ischemic core extension at 24 hours were correlated to bad outcome: $79 \%$ with mRS 3-6 $(P=.002)$ and $88 \%$ with mRS $3-6(P<.001)$, respectively. On multivariate analysis, only recanalization time was significant, with an odds ratio of 1.009 (95\% CI, 1.002-1.016) for each increase of $60 \mathrm{~min}$ utes $(P=.0093)$.

\section{Protocol Deviation}

We included 26 patients with a DWI-ASPECTS $<5$ : 2 younger than 50 years, five $50-59$ years, seven $60-69$ years, eleven $70-79$ years, and one 80 years or older (Fig 3 ). Initially, patients with an ASPECTS lower than 5 should not have been considered for mechanical treatment. Nevertheless, the ASPECTS in an emergency has been, in some cases, underestimated by the team on call. Sometimes they decided to try to recanalize the patient to avoid a malignant infarction; this choice explained a large part of the protocol deviation observed in this series. Each case has been reviewed by 2 neuroradiologists to independently assess the initial ASPECTS and confirm clear protocol deviations. For this population, the mean NIHSS for 70 years and older was 17.24, and the mean time between symptom onset and recanalization was 311 minutes. Stand-alone thrombectomy was received by $58 \%$, and combined procedures, by $42 \%$. The recanalization rate of TICI $2 \mathrm{~b} / 3$ was achieved in $80 \%$, and no difference existed in terms of complications periprocedurally and symptomatic hemorrhagic transformation. Only $10 \%$ of elderly patients (older than 70 ) who were considered to have protocol deviations regarding the initial DWI-MR imaging (ASPECTS <5) achieved a good outcome, whereas $60 \%$ of patients younger than 70 years of age had an mRS $0-2$ at 3 months. Ten patients in the subgroup 70-79 years were considered to have protocol deviations and were all rated mRS 3-6 at 3 months. The ASPECTS for good outcomes at 3 months was 7.4 for 80 years and older versus 6.31 for younger than 50 years, 5.91 for $50-59$ years, 6 for $60-69$ years, and 6.8 for $70-79$ years.

Three patients had NIHSS of $<8$ : 1 in the $50-59$ year group, 1 in the 60-69 year group, and 1 in the 80 years and older group. These 3 patients presented with a symptomatic large-vessel stroke of the ICA. The practitioners on call should have waited for a clinical worsening of the patients before any treatment. Nevertheless, vertigo and dizziness were reported by 1 patient with an intracranial ICA occlusion with an NIHSS score of 5. Because the high risk of major stroke has been proved in the case of symptomatic large-vessel stroke, they decided to treat by a mechanical approach. In the second patient, a symptomatic intracranial carotid bifurcation occlusion was reported with a fluctuating deficit of $5-8$. The patient scored 5 at admission, and despite the threshold of 8 having not been reached, the team decided to proceed with treatment. The 2 patients were fully recanalized with a final $\mathrm{mRS}$ 1 at 3 months. In the third case, the NIHSS score was 7 for an MCA occlusion, and the ASPECTS was 10. The team on call considered a clinical/radiologic mismatch present and started a combined strategy despite the initial NIHSS score of 7.

\section{DISCUSSION}

This study showed a major difference in patient outcome depending on age (Tables 1 and 2). In our study, $50 \%$ of patients were younger than 70 years. In this younger population, thrombectomy efficacy results seemed to be promising, with a good outcome between $58 \%$ and $72 \%$ at 3 months. These results are encouraging, considering that these patients initially had a severe neurologic deficit and a high NIHSS, secondary to a large-vessel occlusion, which is poorly treatable by IV fibrinolysis alone. ${ }^{32-34}$ A large prospective multicenter study of large-vessel occlusion in the anterior circulation treated with the Solitaire device (Covidien) recently reported a favorable outcome (mRS 0-2) in 57.9\% of patients with a mean age of 68.4 years, underlining the importance of age. ${ }^{35}$ In our study, there seemed to be an important cutoff at 70 years of age. Only $19 \%$ of patients older than 80 years had a good outcome at 3 months and $28 \%$ between 70 and 79 years. Loh et $\mathrm{al}^{36}$ also showed that elderly patients were more 
likely to die from their stroke than those younger than 80 years, regardless of recanalization success ( $48 \%$ of mortality versus $15 \%$; OR, 5.5).

These differences among age subgroups are not related to a delayed presentation time for the elderly. The time between onset of symptoms and recanalization was, in our study, roughly equivalent among age classes. Moreover, from a technical point of view, the TICI $2 \mathrm{~b} / 3$ recanalization rate was roughly similar between elderly and younger patients, despite predictably more challenging anatomy in the elderly. The complication rate was in the same range for each age subgroup. Consequently, the huge difference in outcomes between elderly and young patients cannot be solely related to technical issues.

However, physiologic differences may influence this poor outcome in elderly patients. The NIHSS is a well-established initial prognostic factor for intravenous thrombolysis ${ }^{37}$ and endovascular treatment. ${ }^{38,39}$ The mean initial NIHSS for a good outcome at 3 months was 15.74 versus 18.21 for an unfavorable one. For the same level of occlusion and initial DWI-ASPECTS, the mean initial NIHSS was higher in elderly patients ( 18.1 for 70 years and older versus 16.1 for younger than 70 years). A higher NIHSS at entrance for the same level of occlusion could be the direct consequence of a physiologically less effective collateral blood supply and therefore larger penumbra in the elderly. The elderly also have less vascular reserve ${ }^{40,41}$; hence infarct progression may be more rapid and may hence lead to a worse outcome. Furthermore, the elderly have a higher baseline disability before acute stroke and higher rates of in-hospital complications and are more likely to have comorbidities and stroke complications. ${ }^{42,43}$

Initial ASPECTS is an important prognostic criterion closely related to decision-making. Initial infarct size has proved to be a critical determinant of the 3-month functional outcome. ${ }^{44-46}$ Nezu et $\mathrm{al}^{47}$ showed that an ASPECTS $\leq 5$ was related to symptomatic intracerebral hemorrhage and ASPECTS $\geq 7$ corresponded to an mRS score of $0-2$ at 3 months in patients treated with rtPA. In our stroke-management protocol, an ASPECTS $<5$ was therefore initially chosen as an exclusion criterion. Nevertheless, the predictive values of the ASPECTS for a good outcome were very different depending on age subgroups. Our study showed that the 80 years and older group who had good outcomes at 3 months had a mean ASPECTS of 7.4 versus 5.91 for patients $60-69$ years. In addition, $90 \%$ of elderly patients who were considered to have protocol deviations on initial DWI-MR imaging (high initial necrotic core with ASPECTS $<5$ ) demonstrated a bad outcome at 3 months. On the contrary, we observed a good outcome for DWI-MR imaging protocol deviation (ASPECTS $<5$ ) in patients younger than 70 years of age in $60 \%$, suggesting that younger patients may still benefit from acute recanalization despite more extensive initial necrotic cores. Initial MR imaging selection criteria may need to be refined and adapted, depending on the age class, with probably a lower necrotic core volume (initial ASPECTS-DWI) cutoff for elderly patients and, conversely, a higher volume for patients younger than 70 years. DWI lesion volume was not estimated in our study. Using DWI lesion volume could be an interesting work to purchase and ameliorate our results on the ASPECTS evaluation to refine the conclusion. ${ }^{48}$

In our protocol, stand-alone thrombectomy and combined therapy were not randomized. Most interesting, we noticed that there was no significant difference in terms of prognosis in individuals treated by endovascular therapy alone versus combined treatment in each age category. Nevertheless, the synergy between thrombolysis and endovascular therapy was more frequent in young patients due to rare IV contraindications in this subgroup compared with the elderly. Because Davalos et al ${ }^{39}$ demonstrated that combined therapy had better results at 3 months than stand-alone cases ( $66 \%$ of good outcome versus $42 \%$ ), there is a possibility that this synergistic effect of combined treatment, more often missing in the elderly group, also influenced the final results.

\section{Limitations}

One of the major limits of our study is the use of decades to separate our patients, which does not consider physiologic age and baseline health. Comorbidities and life expectancy have to be studied jointly with age in making the decision to treat. Furthermore, thrombectomy has been performed by many different neuroradiologists in our center who all had different rates of learning to manage the procedure. In addition, 14 patients (8.5\%) were lost to follow-up and were not analyzed. These results need further confirmation.

\section{CONCLUSIONS}

Endovascular techniques promise good results in terms of prognosis in patients with an acute stroke secondary to a major vessel occlusion. Patients younger than 70 years benefit the most from acute mechanical recanalization in anterior circulation stroke (mRS 0-2, 62\%; mortality, <10\%). However, in patients older than 70 years, the benefit of mechanical thrombectomy drops (mortality between $26 \%$ and $35 \%$ with only $19 \%-26 \%$ chance of good outcome). Elderly patients usually benefit if their baseline ischemic volume is low in comparison with young patients who may still benefit from acute recanalization despite larger necrotic cores. Stroke-volume thresholds should, therefore, be related and adjusted to the patient's age class.

Disclosures: Alain Bonafé-UNRELATED: Consultancy: Covidien, ${ }^{*}$ Stryker.* Vincent Costalat-UNRELATED: Consultancy: Covidien, Stryker, MicroVention, Codman, Balt; Payment for Lectures (including service On Speakers Bureaus): Stryker, Balt, MicroVention, Codman; Payment for Development of Educational Presentations: Covidien, MicroVention, Stryker; Travel/Accommodations/Meeting Expenses Unrelated to Activities Listed: MicroVention, Sequent, Stryker, Codman. *Money paid to the institution.

\section{REFERENCES}

1. Roger VL, Go AS, Lloyd-Jones DM, et al. Heart disease and stroke statistics: 2011 update: a report from the American Heart Association. Circulation 2011;123:e18-e209

2. Miniño AM, Murphy SL, Xu J, et al. Deaths: final data for 2008. Natl Vital Stat Rep 2011;59:1-126

3. Kelly-Hayes M, Beiser A, Kase CS, et al. The influence of gender and age on disability following ischemic stroke: the Framingham study. J Stroke Cerebrovasc Dis 2003;12:119-26

4. Truelsen T, Piechowski-Jozwiak B, Bonita R, et al. Stroke incidence 
and prevalence in Europe: a review of available data. Eur J Neurol 2006;13:581-98

5. Hacke W, Kaste M, Bluhmki E, et al. Thrombolysis with alteplase 3 to 4.5 hours after acute ischemic stroke. $N$ Engl $J$ Med 2008;359:1317-29

6. Tissue plasminogen activator for acute ischemic stroke: the National Institute of Neurological Disorders and Stroke rt-PA Stroke Study Group. N Engl J Med 1995;333:1581-87

7. Longstreth WT Jr, Katz R, Tirschwell DL, et al. Intravenous tissue plasminogen activator and stroke in the elderly. Am J Emerg Med 2010;28:359-63

8. Berrouschot J, Rother J, Glahn J, et al. Outcome and severe hemorrhagic complications of intravenous thrombolysis with tissue plasminogen activator in very old ( $>$ or $=80$ years) stroke patients. Stroke 2005;36:2421-25

9. Engelter ST, Bonati LH, Lyrer PA. Intravenous thrombolysis in stroke patients of $>$ or $=80$ versus $<80$ years of age: a systematic review across cohort studies. Age Ageing 2006;35:572-80

10. Heuschmann PU, Kolominsky-Rabas PL, Misselwitz B, et al. Predictors of in-hospital mortality and attributable risks of death after ischemic stroke: the German Stroke Registers Study Group. Arch Intern Med 2004;164:1761-68

11. Wardlaw JM, Murray V, Berge E, et al. Recombinant tissue plasminogen activator for acute ischaemic stroke: an updated systematic review and meta-analysis. Lancet 2012;379:2364-72

12. García-Caldentey J, Alonso de Lecinana M, Simal P, et al. Intravenous thrombolytic treatment in the oldest old. Stroke Res Treat 2012;2012:923676

13. Smith WS, Sung G, Saver J, et al. Mechanical thrombectomy for acute ischemic stroke: final results of the Multi MERCI trial. Stroke 2008;39:1205-12

14. Rha JH, Saver JL. The impact of recanalization on ischemic stroke outcome: a meta-analysis. Stroke 2007;38:967-73

15. Mazighi M, Serfaty JM, Labreuche J, et al. Comparison of intravenous alteplase with a combined intravenous-endovascular approach in patients with stroke and confirmed arterial occlusion (RECANALISE study): a prospective cohort study. Lancet Neurol 2009;8:802-09

16. Roth C, Papanagiotou P, Behnke S, et al. Stent-assisted mechanical recanalization for treatment of acute intracerebral artery occlusions. Stroke 2010;41:2559-67

17. Penumbra Pivotal Stroke Trial Investigators. The Penumbra Pivotal Stroke Trial: safety and effectiveness of a new generation of mechanical devices for clot removal in intracranial large vessel occlusive disease. Stroke 2009;40:2761-68

18. Saver JL, Jahan R, Levy EI, et al. Solitaire flow restoration device versus the Merci retriever in patients with acute ischaemic stroke (SWIFT): a randomised, parallel-group, non-inferiority trial. Lancet 2012;380:1241-49

19. Castaño C, Dorado L, Guerrero C, et al. Mechanical thrombectomy with the Solitaire $A B$ device in large artery occlusions of the anterior circulation: a pilot study. Stroke 2010;41:1836-40

20. Costalat V, Machi P, Lobotesis K, et al. Rescue, combined, and stand-alone thrombectomy in the management of large vessel occlusion stroke using the Solitaire device: a prospective 50-patient single-center study: timing, safety, and efficacy. Stroke 2011;42:1929-35

21. Machi P, Costalat V, Lobotesis K, et al. Solitaire FR thrombectomy system: immediate results in $\mathbf{5 6}$ consecutive acute ischemic stroke patients. J Neurointerv Surg 2012;4:62-66

22. Pexman JH, Barber PA, Hill MD, et al. Use of the Alberta Stroke Program Early CT Score (ASPECTS) for assessing CT scans in patients with acute stroke. AJNR Am J Neuroradiol 2001;22:1534-42

23. Barber PA, Demchuk AM, Zhang J, et al. Validity and reliability of a quantitative computed tomography score in predicting outcome of hyperacute stroke before thrombolytic therapy: ASPECTS Study
Group-Alberta Stroke Programme Early CT Score. Lancet 2000; 355:1670-74

24. Butcher KS, Parsons M, MacGregor L, et al. Refining the perfusiondiffusion mismatch hypothesis. Stroke 2005;36:1153-59

25. Wittsack HJ, Ritzl A, Fink GR, et al. MR imaging in acute stroke: diffusion-weighted and perfusion imaging parameters for predicting infarct size. Radiology 2002;222:397-403

26. Hacke W, Kaste M, Fieschi C, et al. Intravenous thrombolysis with recombinant tissue plasminogen activator for acute hemispheric stroke: the European Cooperative Acute Stroke Study (ECASS). JAMA 1995;274:1017-25

27. Hacke W, Kaste M, Fieschi C, et al. Randomised double-blind placebo-controlled trial of thrombolytic therapy with intravenous alteplase in acute ischaemic stroke (ECASS II): Second EuropeanAustralasian Acute Stroke Study Investigators. Lancet 1998;352: 1245-51

28. Furlan A, Higashida R, Wechsler L, et al. Intra-arterial prourokinase for acute ischemic stroke: the PROACT II study-a randomized controlled trial. Prolyse in Acute Cerebral Thromboembolism. JAMA 1999;282:2003-11

29. IMS II Trial Investigators. The Interventional Management of Stroke (IMS) II study. Stroke 2007;38:2127-35

30. Rankin J. Cerebral vascular accidents in patients over the age of 60 . II. Prognosis. Scott Med J 1957;2:200-15

31. van Swieten JC, Koudstaal PJ, Visser MC, et al. Interobserver agreement for the assessment of handicap in stroke patients. Stroke 1988;19:604-07

32. Saqqur M, Uchino K, Demchuk AM, et al. Site of arterial occlusion identified by transcranial Doppler predicts the response to intravenous thrombolysis for stroke. Stroke 2007;38:948-54

33. Linfante I, Llinas RH, Selim M, et al. Clinical and vascular outcome in internal carotid artery versus middle cerebral artery occlusions after intravenous tissue plasminogen activator. Stroke 2002;33: 2066-71

34. Molina CA, Alexandrov AV, Demchuk AM, et al. Improving the predictive accuracy of recanalization on stroke outcome in patients treated with tissue plasminogen activator. Stroke 2004;35:151-56

35. Pereira VM, Gralla J, Davalos A, et al. Prospective, multicenter, single-arm study of mechanical thrombectomy using Solitaire flow restoration in acute ischemic stroke. Stroke 2013;44:2802-07

36. Loh Y, Kim D, Shi ZS, et al. Higher rates of mortality but not morbidity follow intracranial mechanical thrombectomy in the elderly. AJNR Am J Neuroradiol 2010;31:1181-85

37. Adams HP Jr, Davis PH, Leira EC, et al. Baseline NIH Stroke Scale score strongly predicts outcome after stroke: a report of the Trial of Org 10172 in Acute Stroke Treatment (TOAST). Neurology 1999;53: $126-31$

38. Costalat V, Lobotesis $\mathrm{K}$, Machi $\mathrm{P}$, et al. Prognostic factors related to clinical outcome following thrombectomy in ischemic stroke (RECOST study): 50 patients prospective study. Eur J Radiol 2012; 81:4075-82

39. Dávalos A, Pereira VM, Chapot R, et al. Retrospective multicenter study of Solitaire FR for revascularization in the treatment of acute ischemic stroke. Stroke 2012;43:2699-705

40. Epstein SE, Lassance-Soares RM, Faber JE, et al. Effects of aging on the collateral circulation, and therapeutic implications. Circulation 2012;125:3211-19

41. Hecht N, He J, Kremenetskaia I, et al. Cerebral hemodynamic reserve and vascular remodeling in C57/BL6 mice are influenced by age. Stroke 2012;43:3052-62

42. Di Carlo A, Lamassa M, Pracucci G, et al. Stroke in the very old: clinical presentation and determinants of 3-month functional outcome: a European perspective-European BIOMED Study of Stroke Care Group. Stroke 1999;30:2313-19

43. Denti L, Scoditti U, Tonelli C, et al. The poor outcome of ischemic stroke in very old people: a cohort study of its determinants. $\mathrm{J} \mathrm{Am}$ Geriatr Soc 2010;58:12-17

44. Yoo AJ, Chaudhry ZA, Nogueira RG, et al. Infarct volume is a pivotal 
biomarker after intra-arterial stroke therapy. Stroke 2012;43: 1323-30

45. Yoo AJ, Verduzco LA, Schaefer PW, et al. MRI-based selection for intra-arterial stroke therapy: value of pretreatment diffusionweighted imaging lesion volume in selecting patients with acute stroke who will benefit from early recanalization. Stroke 2009; 40:2046-54

46. Sanák D, Nosal V, Horak D, et al. Impact of diffusion-weighted MRI-measured initial cerebral infarction volume on clinical outcome in acute stroke patients with middle cerebral artery occlusion treated by thrombolysis. Neuroradiology 2006;48: 632-39

47. Nezu T, Koga M, Kimura K, et al. Pretreatment ASPECTS on DWI predicts 3-month outcome following rt-PA: SAMURAI rt-PA Registry. Neurology 2010;75:555-61

48. de Margerie-Mellon C, Turc G, Tisserand M, et al. Can DWIASPECTS substitute for lesion volume in acute stroke? Stroke 2013; 44:3565-67 